\title{
Rigid Band Shifts in Two-Dimensional Semiconductors through External Dielectric Screening
}

\author{
Lutz Waldecker $\odot,{ }^{1,2,{ }^{*}, \|}$ Archana Raja $\odot,{ }^{3,4,+, \|}$ Malte Rösner, ${ }^{5,+, \|}$ Christina Steinke, ${ }^{6,7}$ Aaron Bostwick, ${ }^{8}$ Roland J. Koch $\odot,{ }^{8}$ \\ Chris Jozwiak, ${ }^{8}$ Takashi Taniguchi, ${ }^{9}$ Kenji Watanabe $\odot,{ }^{9}$ Eli Rotenberg, ${ }^{8}$ Tim O. Wehling, ${ }^{6,7}$ and Tony F. Heinz $\oplus^{1,2,8}$ \\ ${ }^{1}$ Department of Applied Physics, Stanford University, 348 Via Pueblo Mall, Stanford, California 94305, USA \\ ${ }^{2}$ SLAC National Accelerator Laboratory, Menlo Park, California 94025, USA \\ ${ }^{3}$ Molecular Foundry, Lawrence Berkeley National Laboratory, Berkeley, California 94720, USA \\ ${ }^{4}$ Kavli Energy NanoScience Institute, University of California Berkeley, Berkeley, California 94720, USA \\ ${ }^{5}$ Institute for Molecules and Materials, Radboud University, 6525 AJ Nijmengen, Netherlands \\ ${ }^{6}$ Institute for Theoretical Physics, University of Bremen, Otto-Hahn-Allee 1, 28359 Bremen, Germany \\ ${ }^{7}$ Bremen Center for Computational Material Sciences, University of Bremen, Am Fallturm 1a, 28359 Bremen, Germany \\ ${ }^{8}$ Advanced Light Source, Lawrence Berkeley National Laboratory, Berkeley, California 94720, USA \\ ${ }^{9}$ National Institute for Materials Science, Tsukuba, Ibaraki 305-004, Japan
}

(Received 24 June 2019; published 13 November 2019)

\begin{abstract}
We investigate the effects of external dielectric screening on the electronic dispersion and the band gap in the atomically thin, quasi-two-dimensional (2D) semiconductor $\mathrm{WS}_{2}$ using angle-resolved photoemission and optical spectroscopies, along with first-principles calculations. We find the main effect of increased external dielectric screening to be a reduction of the quasiparticle band gap, with rigid shifts to the bands themselves. Specifically, the band gap of monolayer $\mathrm{WS}_{2}$ is decreased by about $140 \mathrm{meV}$ on a graphite substrate as compared to a hexagonal boron nitride substrate, while the electronic dispersion of $\mathrm{WS}_{2}$ remains unchanged within our experimental precision of $17 \mathrm{meV}$. These essentially rigid shifts of the valence and conduction bands result from the special spatial structure of the changes in the Coulomb potential induced by the dielectric environment of the monolayer.
\end{abstract}

DOI: 10.1103/PhysRevLett.123.206403

In monolayers of atomically thin, quasi-two-dimensional (2D) semiconductors, screening of Coulomb interactions is reduced compared to that present in the corresponding bulk crystals, since electric field lines between charges extend significantly outside the material. As a result, the quasiparticle band gap and exciton binding energies in 2D materials are enhanced by several hundreds of $\mathrm{meV}$ in monolayer transition metal dichalcogenides (TMDCs) [1-8]. By embedding atomically thin materials in different dielectric environments, the Coulomb interaction and therefore the band gap and exciton binding energy can be tuned on the order of a few hundred meV [9-12]. This sensitivity becomes particularly important in vertical heterostructures of 2D materials and enables a noninvasive way of designing nanoscale functionality, such as lateral heterojunctions, through the spatial control of substrate dielectrics $[9,11,13]$.

To exploit the full potential of externally tailoring Coulomb interactions, it is critical to understand the impact of the dielectric environment not only on the band gap and exciton states, but also on the valence- and conductionband dispersions. The dispersion determines properties like charge carrier effective masses and energy differences between different valleys within the Brillouin zone. To date, experimental studies of dielectric engineering have mainly focused on optical spectroscopy, scanning tunneling microscopy, or electronic transport measurements of TMDC monolayers, which do not give direct access to the electronic dispersion. In general, however, perturbations to a material do not have the same effect on electronic states of different orbital character and can be expected to modify the band structure in different parts of the Brillouin zone differently.

Here, through a combination of experiment and theory, we provide a comprehensive picture of the consequences of dielectric screening on the band structure of 2D semiconductors. By combining angle-resolved photoemission spectroscopy with micrometer spatial resolution ( $\mu$ ARPES) and optical spectroscopy of the exciton states of monolayer $\mathrm{WS}_{2}$ on different substrates, we find that the predominant effect of external dielectric screening is a band-gap renormalization through a rigid shift of the occupied and unoccupied bands relative to each other. These rigid shifts are the result of the spatial structure of the changes in the Coulomb potential induced by the dielectric environment, which we elucidate with the aid of ab initio $G \Delta W$ calculations.

In our experiment, monolayers of $\mathrm{WS}_{2}$ were exfoliated from bulk crystals and transferred so that they partially cover two different substrates, hexagonal boron nitride (hBN), a wide band-gap insulator, and graphite, a semimetal (for 
(a)

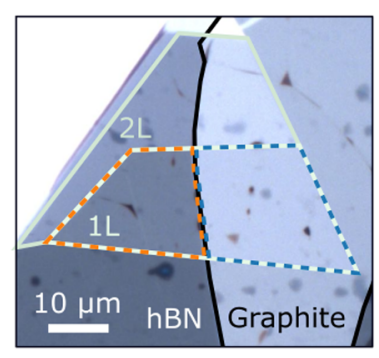

(b)

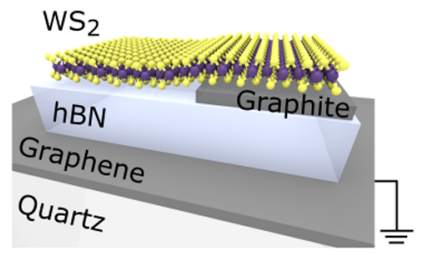

(d)

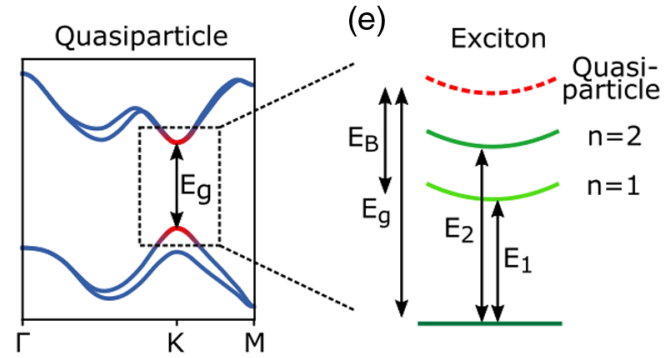

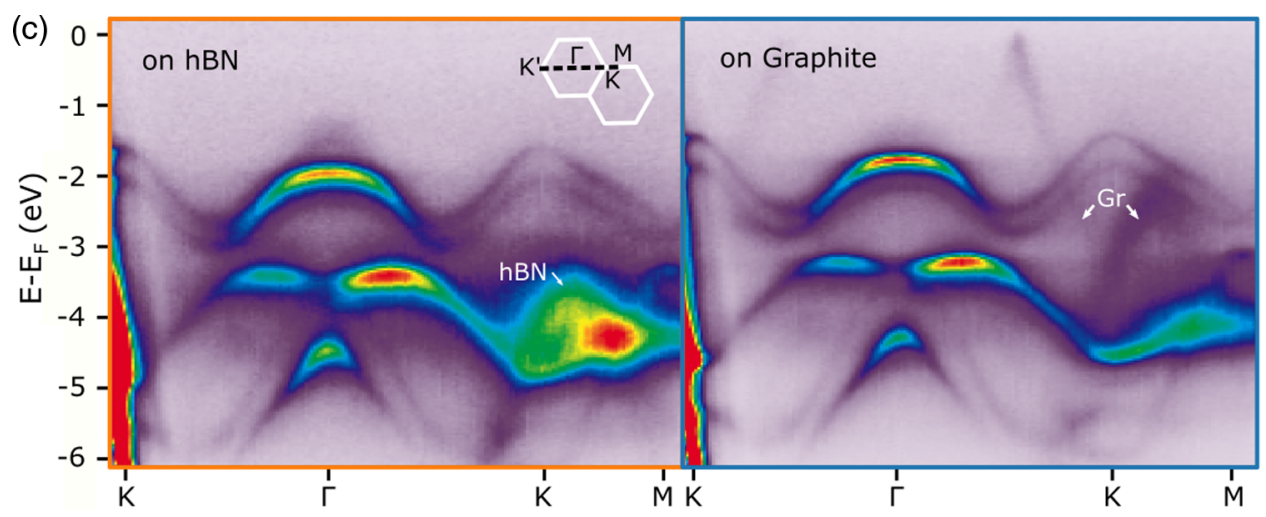

(f)

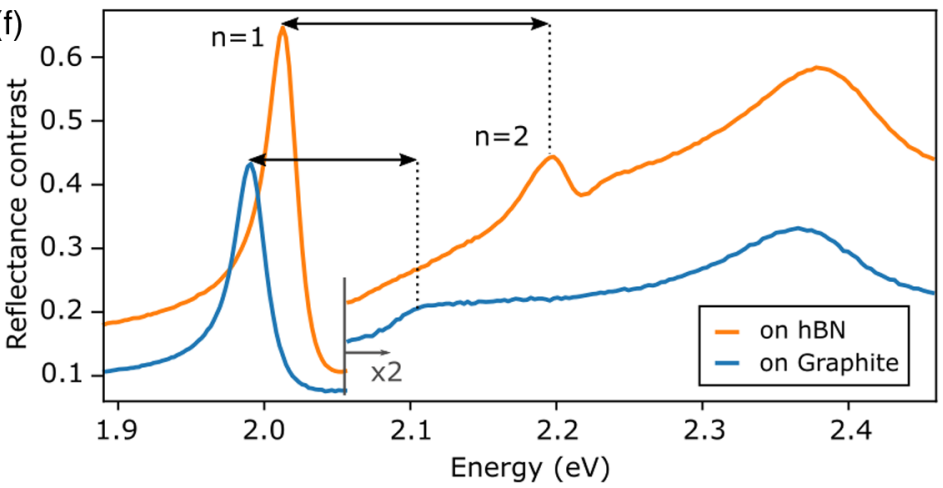

FIG. 1. (a) Optical micrograph of monolayer $\mathrm{WS}_{2}$ (dotted white line) straddling distinct dielectric environments of $\mathrm{hBN}$ (orange) and graphite (blue). (b) Sample geometry on a transparent conductive substrate, enabling both optical spectroscopy and ARPES measurements. (c) Room-temperature photoemission intensity maps along $K^{\prime}-\Gamma-K-M$ (see the inset) of monolayer $\mathrm{WS}_{2}$ on $\mathrm{hBN}$ and on graphite. (d) Sketch of the band structure, showing the direct band gap. The quasiparticle states around the $K$ point that form the $A$ exciton transition are highlighted in red. (e) Schematic of exciton ground and excited state transitions, showing the relationship between exciton transition energies, exciton binding energy $E_{B}$, and band gap $E_{g}$ corresponding to the $K\left(K^{\prime}\right)$ point transition. (f) Roomtemperature reflectance contrast spectrum of monolayer $\mathrm{WS}_{2}$ on $\mathrm{hBN}$ (orange) and on graphite (blue), measured on the same sample areas as the photoemission maps in (c).

experimental details, see Supplemental Material [14]). An optical micrograph of a typical sample and a schematic of the sample geometry are shown in Figs. 1(a) and 1(b).

Using $\mu$-ARPES, we measure the valence-band dispersion and the separation to shallow core levels, including the $\mathrm{W}_{4 f}$ level. Two examples of room-temperature photoemission intensity maps of the valence bands of $\mathrm{WS}_{2}$ in the $K^{\prime}-\Gamma-K-M$ direction are presented in Fig. 1(c). Signatures of the respective substrates appear in both spectra, such as the $\pi$ band of hBN and replicas of graphite bands extending to the Fermi energy $E_{F}$. However, no signs of hybridization between $\mathrm{WS}_{2}$ and the respective substrate bands are observed.

By measuring the exciton states on the same samples, we obtain information on quasiparticle band gaps at the $K$ points [Fig. 1(d)]. Typical reflectance contrast spectra, approximately proportional to the monolayer absorption, are shown in Fig. 1(f). As the oscillator strength in 2D semiconductors resides mainly in their excitonic absorption features, a series of prominent peaks is seen in the spectra. We identify the two lowest lying features as the $n=1$ and $n=2$ states of the $A$ exciton transition [5]. While the quasiparticle band gap is not directly accessible, it scales with the separation between the $n=1$ and $n=2$ exciton states, $\Delta_{12}$ [Fig. 1(e)] $[5,11,15]$. The exciton binding energy and the quasiparticle band gap are sensitive to dielectric screening from the immediate environment, which is reflected in both the shift of the exciton peaks and, more importantly, the reduction of the energy separation between the $n=1$ and $n=2$ states [11]. The comparable linewidths of the $n=1$ and $n=2$ states indicate a high-quality heterostructure interface [16]. The lack of charged exciton signatures in the optical spectra suggests low doping levels [17], consistent with the ARPES data in which the Fermi level is observed within the band gap.

We now analyze the experimentally measured dispersion and the band-gap renormalization in conjunction with firstprinciples calculations. We calculate the band structure of a freestanding $\mathrm{WS}_{2}$ monolayer in the $G W$ approximation, from which the band gap in a vacuum is obtained in good agreement with Ref. [18] (see [14] for details). The change in the band gap is then calculated using a combination of the Wannier function continuum electrostatics [19] and $G \Delta W$ approaches $[20,21] . \Delta W$ is the externally induced 

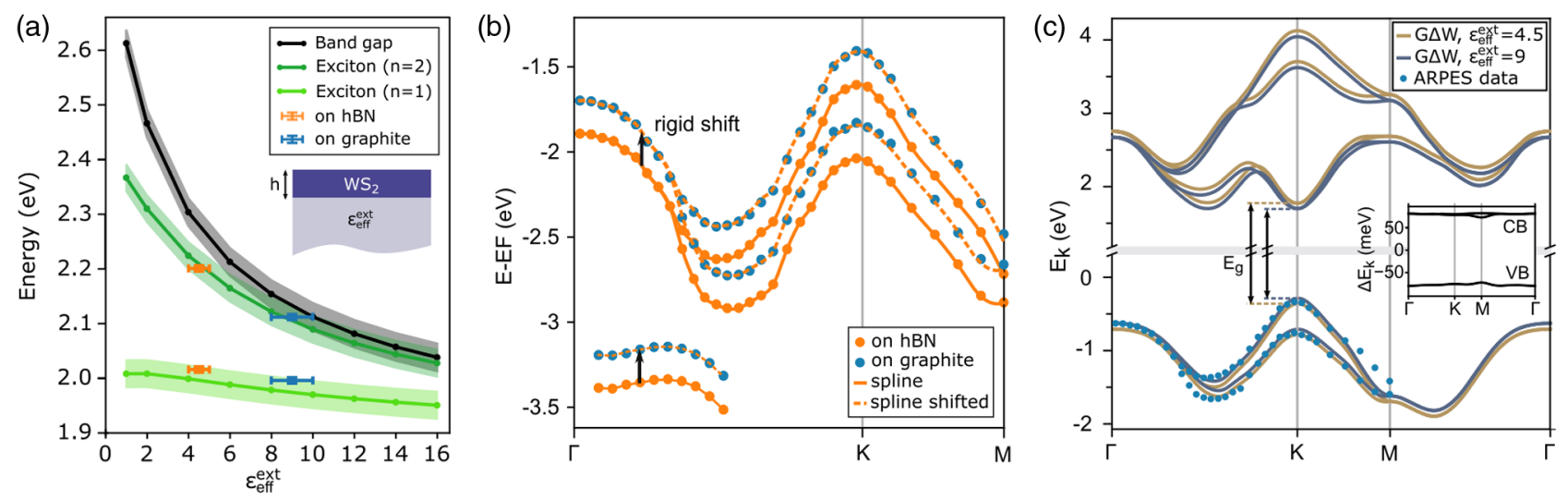

FIG. 2. (a) Calculated quasiparticle band gap and $n=1$ and $n=2$ exciton energies as a function of $\varepsilon_{\text {eff }}^{\text {ext }}$ (lines) and experimental values of the exciton energies on hBN and graphite (markers). (b) Experimental valence-band positions on hBN (orange points) and graphite (blue points) along the $\Gamma-K-M$ direction from the ARPES measurements. A quadratic spline through the data points on hBN is shown in solid orange, which can be rigidly shifted by $0.18 \mathrm{eV}$ to overlay the data points on graphite (dashed orange line). (c) Calculated valence and lowest conduction bands (lines) and experimental results (circles) for the valence bands. The experimental data have been aligned to the calculated curves at the $\Gamma$ points. Inset: Difference of the calculated dispersion between the conduction and valence bands of $\mathrm{WS}_{2}$ supported on substrates with $\varepsilon_{\mathrm{eff}}^{\mathrm{ext}}=4.5$ and $\varepsilon_{\mathrm{eff}}^{\mathrm{ext}}=9$.

change to the Coulomb potential resulting from a semiinfinite dielectric substrate described by an effective dielectric constant $\varepsilon_{\text {eff }}^{\text {ext }}$ acting on the $\mathrm{WS}_{2}$ monolayer of thickness $h=6.162 \AA$ [see Fig. 2(a), inset]. In this way, we reduce the complex dielectric function of the substrates to an effective screening constant $\varepsilon_{\text {eff }}^{\text {ext }}$, independent of momentum and frequency.

The calculated change of the quasiparticle band gap, i.e., the difference between the valence-band maximum and the conduction-band minimum at the $K$ points, is shown in Fig. 2(a). To compare this result to the measured exciton energies, we further solve the Wannier equation for the screened potentials (i.e., as a function of $\varepsilon_{\text {eff }}^{\text {ext }}$ ) and obtain the binding energies of $n=1$ and $n=2$ exciton states (similar to Ref. [15]; for details see [14]). We find good agreement between experimental and calculated exciton positions for $\varepsilon_{\text {eff }}^{\text {ext }}=4.5$ and $\varepsilon_{\text {eff }}^{\text {ext }}=9$ for $\mathrm{hBN}$ and graphite, respectively [see Fig. 2(a)]. These values are in reasonable agreement with previously reported values of dielectric constants and exciton binding energies on hBN [22]. We note that this mapping of the external dielectric function $\varepsilon^{\text {ext }}(q, \omega) \mapsto$ $\varepsilon_{\text {eff }}^{\text {ext }}$ to an effective external dielectric constant using $\Delta_{12}$ renders $\varepsilon_{\text {eff }}^{\text {ext }}$ specific for $\mathrm{WS}_{2}$. The calculated band-gap renormalization upon changing $\varepsilon_{\text {eff }}^{\text {ext }}=4.5$ to $\varepsilon_{\text {eff }}^{\text {ext }}=9$ is found to be $140 \mathrm{meV}$. We thus predict the band gap of monolayer $\mathrm{WS}_{2}$ on $\mathrm{hBN}$ to be approximately $140 \mathrm{meV}$ larger than on graphite.

To examine experimentally how the external dielectric screening affects the band dispersion, we determine the band energies from the ARPES data in Fig. 1(c) by fitting energy distribution curves of the valence bands at each recorded parallel momentum $k_{x}$ and accounting for detector distortions (see [14]). Intriguingly, a quadratic spline through the data points on hBN can be rigidly shifted to overlay the data points on graphite within our experimental uncertainty of $17 \mathrm{meV}$, determined by the standard deviation of the difference between the two datasets. In particular, the relative alignment of the $K$ points with respect to $\Gamma$ is determined as $280(280) \pm 10 \mathrm{meV}$ on hBN (graphite) and the spin-orbit splitting at the $K$ points [23] is $440(430) \pm 20 \mathrm{meV}$. The effective masses in the valence bands are inferred from quadratic fits to be $2.45(2.55) \pm$ $0.05 m_{e}$ at $\Gamma$ and $0.48(0.48) \pm 0.05 m_{e}$ in the upper and $0.64(0.78) \pm 0.1 m_{e}$ in the lower valence band at the $K$ points on $\mathrm{hBN}$ (graphite). Additionally, we observe that the energy difference between $\mathrm{W}_{4 f}$ core level states and the valence bands remain the same on both substrates, within experimental uncertainty (see [14]).

The calculated valence- and conduction-band dispersions for the two values of $\varepsilon_{\text {eff }}^{\text {ext }}$ are shown in Fig. 2(c) together with the experimental data points on graphite. For a direct comparison, we align the experimental bands to the $G \Delta W$ calculations at their $\Gamma$-point energies, since the band alignment of different regions can depend on factors such as doping or the difference in work function of the substrates [24]. The calculated curves closely follow the measured dispersion, with small deviations roughly halfway between $\Gamma$ and $K$ as well as near $M$. These discrepancies may arise from the difficulty of fitting two bands with a small energy separation, and in this region of strong orbital hybridization we expect the calculations to be particularly sensitive to small errors in lattice relaxation.

From our calculations of the dispersion, it is clear that the main effect of the external dielectric screening is a rigid shift of occupied and unoccupied bands, as also calculated in Ref. [21]. The shift is symmetric in the valence and conduction bands, which is to first order intrinsic to the 2D slab geometry, as discussed below. The change in 
dispersion induced by the substrate is shown for the conduction and valence bands in the inset in Fig. 2(c), where we plot the difference between the band energies $\Delta E(k)=E_{\varepsilon_{\text {eff }}^{\text {ext }}=4.5}(k)-E_{\varepsilon_{\text {eff }}^{\text {ext }}=9}(k)$. In the calculations, the deviations from a rigid shift are less than $5 \mathrm{meV}$ across the Brillouin zone, which is consistent with our experimental bound on the change in the valence-band dispersion.

These essentially rigid shifts in the bands can be understood from the change of the Coulomb interaction profile $\Delta W_{\varepsilon_{\text {eff }}^{\text {ext }}}(\rho)=W_{\varepsilon_{\text {eff }}}(\rho)-W_{V}(\rho)$ and its effects on the $G \Delta W$ band structure as resulting from the screening environment with effective dielectric constant $\varepsilon_{\text {eff }}^{\text {ext }}$ compared with the freestanding layer in a vacuum ( $V$, i.e., $\varepsilon_{\text {eff }}^{\text {ext }}=1$ ) (see [14]). In Fig. 3(a), we show examples of $\Delta W_{\varepsilon_{\text {eff }}^{\text {ext }}=4.5}$ and $\Delta W_{\varepsilon_{\text {eff }}^{\text {ext }}=9}$ as functions of the real-space coordinate $\rho$ (inset) and the momentum transfer $q$. Since $W_{V}$ is always larger than $W_{\varepsilon_{\text {exf }}^{\text {ext }}}, \Delta W_{\varepsilon_{\text {eff }}^{\text {ext }}}$ is by definition negative. In real space, we find nearly constant potential profiles for $\rho<10 \AA$. At larger $\rho$, the change in potential approaches zero. This behavior of $\Delta W_{\varepsilon_{\text {exf }}^{\text {ext }}}(\rho)$ results from the nonlocal screening properties of the $2 \mathrm{D}$ slab with finite height $h$. In the case of purely local screening, we expect $\Delta W_{\varepsilon}^{\text {loc }}(\rho) \propto(1 / \varepsilon \rho)-(1 / v)=[(1-\varepsilon) / \varepsilon \rho]$ to diverge for small $\rho$. Here, however, the two dielectric interfaces (top and bottom side of the $\mathrm{WS}_{2}$ layer) are separated by $\pm h / 2$ from the center of the slab and create an alternating infinite series of image charges localized at distances $h \gtrsim 6 \AA$. The corresponding contributions to $\Delta W_{\varepsilon_{\text {eff }}^{\text {ext }}}(\rho)$ are of the form $\propto\left(1 / \sqrt{h^{2}+\rho^{2}}\right)[15]$ and, thus, flat at small $\rho$. Therefore, it is necessary to fully take the effective height $h$ into account. The well-known approximation of the Keldysh potential $W(\rho) \propto \alpha^{-1}\left[H_{0}(\rho / \alpha)-J_{0}(\rho / \alpha)\right]$ [25], which is valid only for $\rho \gg h$, is therefore not capable of accurately describing this particular change in the interaction profile.

The flat interaction profile $\Delta W_{\varepsilon_{\text {eff }}^{\text {eft }}}(\rho)$ in real space translates to a strongly peaked profile in momentum space, vanishing for $q \gtrsim 0.15 \AA^{-1}$. For the following analysis, we can thus approximate $\Delta W_{\varepsilon_{\text {eff }}^{\text {ext }}}(\rho) \approx \Delta W_{\varepsilon_{\text {exf }}^{\text {ext }}}(\rho=0)=$ $\gamma_{\varepsilon_{\text {exf }}^{\text {ext }}}$ and $\Delta W_{\varepsilon_{\text {eff }}^{\text {ext }}}(q) \approx \gamma_{\varepsilon_{\text {eff }}^{\text {ext }}} \delta(q)$. Importantly, this type of interaction does not distinguish between different orbital characters and cannot cause any interband scattering.

In this case, the electronic self-energy $\Sigma_{G \Delta W}$, which describes the changes in the electronic quasiparticle dispersions of the $\mathrm{WS}_{2}$ layer due to changes in the external dielectric screening, greatly simplifies in the $G \Delta W$ approximation. For electrons in band $\lambda$ with momentum $k$, it reads

$$
\Sigma_{G \Delta W}^{\lambda}(k, \omega)=\frac{i}{2 \pi} \int d q^{2} \int d \omega^{\prime} \frac{\Delta W_{\varepsilon_{\text {eff }}^{\mathrm{ext}}}\left(q, \omega^{\prime}\right)}{\omega+\omega^{\prime}+i \delta-E_{k-q}^{\lambda}} .
$$

In the static Coulomb-hole plus screened-exchange (COHSEX) approximation, this self-energy $\Sigma_{G \Delta W}$ can be split into two terms resulting from poles in $G$ and in $\Delta W$, yielding $\Sigma_{\mathrm{SEX}}^{\lambda}(k) \approx-\gamma_{\varepsilon_{\text {eff }}^{\text {exf }}} n_{F}\left(E_{k}^{\lambda}\right)$ and $\Sigma_{\mathrm{COH}}^{\lambda}(k) \approx\left(\gamma_{\varepsilon_{\text {eff }}^{\text {ext }}} / 2\right)$, respectively, where $n_{F}$ is the Fermi function. The SEX part shifts only occupied states up in energy, and the $\mathrm{COH}$ terms shifts all bands down by $\gamma_{\varepsilon_{\text {eff }}^{\text {ext }}} / 2$. These self-energies are independent of $k$ for completely filled (empty) valence (conduction) bands, since the Fermi functions depend only on band index $\lambda$. The quasiparticle dispersions under the influence of external dielectric screening then read

$$
E_{k, \varepsilon_{\text {eff }}^{\text {ext }}}^{\lambda}=E_{k, e_{\text {eff }}^{\text {ext }}=1}^{\lambda}+\gamma_{\varepsilon_{\text {eff }}^{\text {ext }}}\left[n_{F}\left(E_{k, \varepsilon_{\text {eff }}^{\text {ext }}=1}^{\lambda}\right)-\frac{1}{2}\right]
$$

The bands thus shift as a whole, with no change in dispersion. The band gap is symmetrically reduced by $\gamma_{\varepsilon_{\text {eff }}}$, equally for all
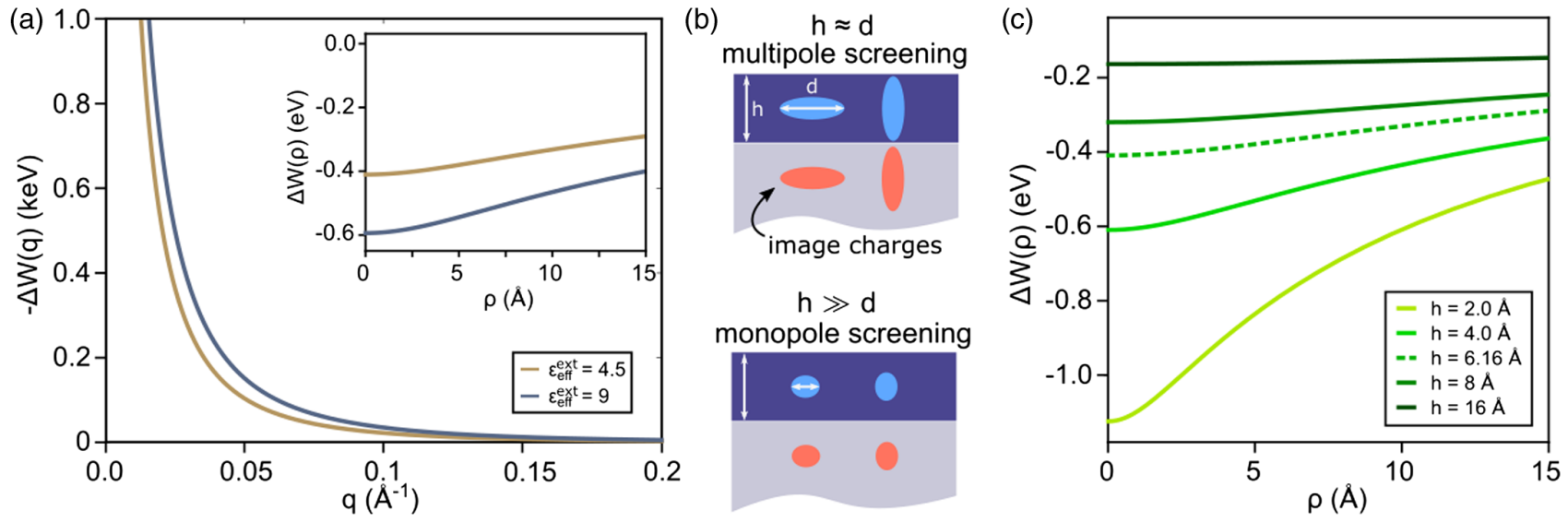

FIG. 3. (a) Change in the Coulomb potential induced by the external dielectric environment, $\Delta W$, as a function of momentum-transfer and real-space separation (inset). (b) Multipole vs monopole screening due to image charges in the substrate for different ratios of layer heights $h$ to spatial orbital extent $d$. For $h \gg d$, monopole screening dominates, and bands are shifted rigidly. (c) Change of the Coulomb potential for different layer thicknesses $h$ and $\varepsilon_{\mathrm{eff}}^{\mathrm{ext}}=4.5$. For small $h$, the change shows a pronounced spatial dependence, which can cause asymmetric band shifts. 
momenta $k$. Experimentally, we observe these rigid shifts down to the core levels $\mathrm{W}_{4 f}$ (see [14]) and also find them in our full COHSEX calculations, using the full orbital-dependent $\Delta W^{\alpha \beta}(q)$ presented in Fig. 2. We note that Cho and Berkelbach have described a similar effect for the band gap at the $K$ point [15]. Here, we prove its validity throughout both the whole Brillouin zone and the full band structure, yielding an analog to molecular level shifts in solvent environments or on surfaces [26,27].

In order to change the band shapes or to induce asymmetric band shifts, significant deviations from the approximation $\Delta W_{\varepsilon_{\text {eff }}^{\text {ext }}}(q) \approx \gamma_{\varepsilon_{\text {eff }}^{\text {exf }}} \delta(q)$ are needed. Thus, either $\gamma_{\varepsilon_{\text {eff }}^{\text {ext }}}$ must become orbital dependent, or $\Delta W_{\varepsilon_{\text {eff }}}(q) \propto$ $\delta(q)$ must break down. This is controlled by the ratio between the effective $\mathrm{WS}_{2}$ thickness $h$ and the spatial extent $d$ of the orbitals. For example, if $h \ll d$, the multipole screening by the image charges differentiates between different orbital characters [see Fig. 3(b)], and $\gamma_{\varepsilon_{\text {eff }}^{\text {ext }}}^{\alpha \beta}$ becomes orbital dependent. Also, for small $h$ the change to the Coulomb potential $\Delta W_{\varepsilon_{\text {eff }}^{\text {ext }}}(\rho)$ starts to show a spatial structure [see Fig. 3(c)], and $\Delta W_{\varepsilon_{\text {exf }}^{\text {exf }}}(q) \propto \delta(q)$ becomes inaccurate. Thus, by reducing the effective height or increasing the spatial extent of the orbitals, changes in the band dispersion may occur. In the case of the TMDCs, the transition metal $d$ orbitals are "shielded" by the surrounding chalcogen atoms, which increases the effective height and reduces non-rigid-shift effects. In effectively thinner materials with multiorbital band-edge character, these effects could, however, become stronger.

Thus, the validity of the approximation $\Delta W_{\varepsilon_{\text {eff }}^{\text {ext }}}(q) \approx$ $\gamma_{\varepsilon_{\text {eff }}^{\text {exf }}} \delta(q)$ is an intrinsic property of the monolayer and certainly holds for $\mathrm{WS}_{2}$. We therefore do not expect any deviations from the rigid changes to the band structure even if the external dielectric screening shows a significant frequency dependence, as in the case of graphite [28]. As shown above, there are indeed no additional changes to the experimentally measured valence bands of monolayer $\mathrm{WS}_{2}$ on graphite [Fig. 2(b)], and our static theory adequately describes the experimental dispersion [Fig. 2(c)].

In conclusion, our combined experimental and theoretical studies show that we induce a rigid shift of the valence and conduction bands in monolayer $\mathrm{WS}_{2}$ by engineering the dielectric environment. The nonlocal nature of the dielectric screening leads to almost constant changes of the Coulomb potential in $\mathrm{WS}_{2}$, which translates to a symmetric opening and closing of the band gap. This mechanism is consistent with recently reported data from transport measurements across a similar dielectrically engineered lateral heterojunction [13]. The observed rigid shifts stand in contrast to other methods of band-gap engineering, such as ion doping, in which the upper valence band at $K$ is modified and the spin splitting of the bands increases [29], or the application of strain, which results in a change of the relative alignment of different valleys [30,31]. Our results thus establish a simple physical picture for the influence of surrounding dielectric media on the electronic properties of 2D semiconductors, necessary both for a complete analysis of the properties of stacked 2D materials and for the development of Coulomb engineering as a noninvasive approach to lateral patterning of 2D systems.

The authors thank Simone Latini for helpful comments about solving the 2D Schrödinger equation, Merzuk Kaltak for sharing his (c)RPA implementation [32], and Alexey Chernikov and Jonah Haber for detailed suggestions about the manuscript. The spectroscopic studies were supported at SLAC by the U.S. Department of Energy (DOE), Office of Science, Basic Sciences, Materials Sciences and Engineering Division, under Contract No. DE-AC0276 SF00515 for analysis, by the Gordon and Betty Moore Foundations EPiQS Initiative through Grant No. GBMF4545 for measurements at Stanford, and by the National Science Foundation under Grant No. DMR1708457 for sample preparation. L. W. and M. R. acknowledge support by the Alexander von Humboldt Foundation. A. R. gratefully acknowledges funding through the Heising-Simons Junior Fellowship within the Kavli Energy NanoScience Institute at the University of California, Berkeley. T. O. W. and C. S. acknowledge support by the European Graphene Flagship and DFG via GRK 2247. K. W. and T. T. acknowledge support from the Elemental Strategy Initiative conducted by the MEXT, Japan, A3 Foresight by JSPS and the CREST (JPMJCR15F3), JST. This research used resources of the Advanced Light Source, which is a DOE Office of Science User Facility under Contract No. DE-AC02-05CH11231.

*waldecker@stanford.edu

†araja@lbl.gov

*m.roesner@science.ru.nl

\$tony.heinz@stanford.edu

"These authors contributed equally to this work.

[1] A. Ramasubramaniam, Phys. Rev. B 86, 115409 (2012).

[2] T. C. Berkelbach, M. S. Hybertsen, and D. R. Reichman, Phys. Rev. B 88, 045318 (2013).

[3] D. Y. Qiu, F. H. da Jornada, and S. G. Louie, Phys. Rev. Lett. 111, 216805 (2013).

[4] K. He, N. Kumar, L. Zhao, Z. Wang, K. F. Mak, H. Zhao, and J. Shan, Phys. Rev. Lett. 113, 026803 (2014).

[5] A. Chernikov, T. C. Berkelbach, H. M. Hill, A. Rigosi, Y. Li, O. B. Aslan, D. R. Reichman, M. S. Hybertsen, and T. F. Heinz, Phys. Rev. Lett. 113, 076802 (2014).

[6] M. M. Ugeda, A. J. Bradley, S. F. Shi, F. H. Da Jornada, Y. Zhang, D. Y. Qiu, W. Ruan, S. K. Mo, Z. Hussain, Z. X. Shen, F. Wang, S. G. Louie, and M. F. Crommie, Nat. Mater. 13, 1091 (2014).

[7] Z. Ye, T. Cao, K. O’Brien, H. Zhu, X. Yin, Y. Wang, S. G. Louie, and X. Zhang, Nature (London) 513, 214 (2014). 
[8] G. Wang, A. Chernikov, M. M. Glazov, T. F. Heinz, X. Marie, T. Amand, and B. Urbaszek, Rev. Mod. Phys. 90, 021001 (2018).

[9] M. Rösner, C. Steinke, M. Lorke, C. Gies, F. Jahnke, and T. O. Wehling, Nano Lett. 16, 2322 (2016).

[10] A. V. Stier, N. P. Wilson, G. Clark, X. Xu, and S. A. Crooker, Nano Lett. 16, 7054 (2016).

[11] A. Raja, A. Chaves, J. Yu, G. Arefe, H. M. Hill, A. F. Rigosi, T. C. Berkelbach, P. Nagler, C. Schüller, T. Korn, C. Nuckolls, J. Hone, L. E. Brus, T. F. Heinz, D. R. Reichman, and A. Chernikov, Nat. Commun. 8, 15251 (2017).

[12] S. Park, N. Mutz, T. Schultz, S. Blumstengel, A. Han, A. Aljarb, L.-J. Li, E. J. W. List-Kratochvil, P. Amsalem, and N. Koch, 2D Mater. 5, 025003 (2018).

[13] M. I. B. Utama, H. Kleemann, W. Zhao, C. S. Ong, F. H. da Jornada, D. Y. Qiu, H. Cai, H. Li, R. Kou, S. Zhao, S. Wang, K. Watanabe, T. Taniguchi, S. Tongay, A. Zettl, S. G. Louie, and F. Wang, Natl. Electron. 2, 60 (2019).

[14] See Supplemental Material at http://link.aps.org/ supplemental/10.1103/PhysRevLett.123.206403 for details on the experiments and calculations.

[15] Y. Cho and T. C. Berkelbach, Phys. Rev. B 97, 041409(R) (2018).

[16] A. Raja, L. Waldecker, J. Zipfel, Y. Cho, S. Brem, J. D. Ziegler, M. Kulig, T. Taniguchi, K. Watanabe, E. Malic et al., Nat. Nanotechnol. 14, 832 (2019).

[17] K. F. Mak, K. He, C. Lee, G. H. Lee, J. Hone, T. F. Heinz, and J. Shan, Nat. Mater. 12, 207 (2013).

[18] S. Haastrup, M. Strange, M. Pandey, T. Deilmann, P. S. Schmidt, N. F. Hinsche, M. N. Gjerding, D. Torelli, P. M. Larsen, A. C. Riis-Jensen, J. Gath, K. W. Jacobsen, J. J. Mortensen, T. Olsen, and K. S. Thygesen, 2D Mater. 5, 042002 (2018).
[19] M. Rösner, E. Şaşığlu, C. Friedrich, S. Blügel, and T. O. Wehling, Phys. Rev. B 92, 085102 (2015).

[20] M. Rohlfing, Phys. Rev. B 82, 205127 (2010).

[21] K. T. Winther and K. S. Thygesen, 2D Mater. 4, 025059 (2017).

[22] K. Andersen, S. Latini, and K. S. Thygesen, Nano Lett. 15, 4616 (2015).

[23] Z. Y. Zhu, Y. C. Cheng, and U. Schwingenschlogl, Phys. Rev. B 84, 153402 (2011).

[24] S. Ulstrup, C. E. Giusca, J. A. Miwa, C. E. Sanders, A. Browning, P. Dudin, C. Cacho, O. Kazakova, D. K. Gaskill, R. L. Myers-Ward, T. Zhang, M. Terrones, and P. Hofmann, Nat. Commun. 10, 3283 (2019).

[25] P. Cudazzo, I. V. Tokatly, and A. Rubio, Phys. Rev. B 84, 085406 (2011).

[26] J. B. Neaton, M. S. Hybertsen, and S. G. Louie, Phys. Rev. Lett. 97, 216405 (2006).

[27] K. S. Thygesen and A. Rubio, Phys. Rev. Lett. 102, 046802 (2009).

[28] M. F. Lin, C. S. Huang, and D. S. Chuu, Phys. Rev. B 55, 13961 (1997).

[29] J. Katoch, S. Ulstrup, R. J. Koch, S. Moser, K. M. McCreary, S. Singh, J. Xu, B. T. Jonker, R. K. Kawakami, A. Bostwick, E. Rotenberg, and C. Jozwiak, Nat. Phys. 14, 355 (2018).

[30] W. S. Yun, S. W. Han, S. C. Hong, I. G. Kim, and J. D. Lee, Phys. Rev. B 85, 033305 (2012).

[31] H. J. Conley, B. Wang, J. I. Ziegler, R. F. Haglund, S. T. Pantelides, and K. I. Bolotin, Nano Lett. 13, 3626 (2013).

[32] M. Kaltak, Merging GW with DMFT, Phd thesis, University of Vienna, (2015). 